Aims To provide emotional support to the statutory carers and health care workers of Inverclyde working throughout the pandemic, offering:

- Confidential group sessions for service managers and teams to explore the impact of working during the pandemic and supporting distressed staff. Exploring impacts on them as individuals outside of work and enhancing resilience.

- Individual support sessions as required, similar to either counselling or clinical supervision as required.

- Availability of spiritually focused sessions for individuals or groups with a focus on enhancing resilience.

- Support of hospice staff with regular information and support around wellbeing and resilience including self-hypnosis training for relaxation, online Pilates and one-to-one support as required.

Methods

- Phase 1 (March-May 2020) Identified support to be offered. Funding secured from the BIG Lottery.

- Phase 2 (May-June 2020): Recruitment and training of sessional staff.

- Phase 3 (September 2020 onwards): Launch and advertisement of service, provision of support.

Results Uptake was initially slower than thought, however, referrals grew steadily with the onset of the second lockdown. Demand for support continues to rise exponentially as the impact of the pandemic takes its toll on carers and healthcare workers.

Conclusion The need for carers and healthcare worker support is as important now as it was in the initial stages of the pandemic, possibly more so. Community need is greater now than ever and requests for help from care and healthcare services are rising rapidly. This is taking a toll on workers who have not yet processed what they themselves have been through throughout this pandemic.

\section{P-183 A LONGITUDINAL SURVEY OF STAFF WELL-BEING AT A HOSPICE DURING THE COVID-19 PANDEMIC}

${ }^{1}$ Rachel Perry, ${ }^{2}$ John MacArtney. ${ }^{1}$ Marie Curie, West Midlands, UK; ${ }^{2}$ University of Warwick, Coventry, UK

\subsection{6/spcare-2021-Hospice. 199}

Background In April 2020 we undertook a survey of staff wellbeing to gain a better understanding of the effects of rapid and substantial change upon all staff during the COVID-19 pandemic. In response to staff feedback to the survey report, we repeated the survey 12 months later to identify some of the longer-term issues related to working through the pandemic.

Aim The aim of this evaluation was to identify opportunities to support staff in their work and their wellbeing during the COVID-19 pandemic.

Methods Design. The survey collected basic demographic information and asked six open-ended questions designed to elicit further detail about staff experience and understanding of rapid change that had been identified as part of the stakeholder engagement.

Setting and recruitment. We invited non-clinical and clinical staff from all departments and teams within a West Midlands hospice.
Analysis. We used a codebook thematic approach (Braun, Clarke, Hayfield, et al., 2019) to identify: (1) descriptive themes to provide a summary of how staff were managing with the rapid change and any shared issues addressing their wellbeing; and, (2) key points of action that could be taken to support staff further.

Findings Staff reported feelings of duty, pride and working together for a common cause and this sense of purpose was also evident in the second survey. Staff initially described feelings of anxiety and frustration, and a year into the pandemic staff are feeling exhausted, challenged, sad, stressed, tired, isolated, overwhelmed, and pressured. Staff reported making significant personal efforts to manage the uncertainties the semi-permanent exceptional working conditions bring to work and home life.

Outcomes and impact The researchers presented the findings to senior management, who have agreed to initiate a programme of support and open communication with staff.

\section{COVID-19; HOPE, COMPASSION, HUMANITY, SHARING MORAL DISTRESS AND CAPTURING POST TRAUMATIC GROWTH}

Emma Louise Shaw, Rachel Morris, Lynda Finney. Marie Curie, Liverpool, UK

10.1136/spcare-2021-Hospice.200

Aims Our project aimed to address staff wellbeing and explore, as a hospice team, some emotional challenges presented by the COVID-19 pandemic. Difficult decision making has caused moral distress amongst staff; it is essential to address this in order to prevent overwhelming trauma, promote mental resilience and foster hope.

Methods Providing a safe environment was necessary for staff to share their feelings. Under the theme of 'Let it Go!', for one week staff were asked to write down their feelings anonymously and take a treat thanking them. An overwhelming response demonstrated the pressure placed on people's feelings of safety and emotional wellbeing. Hospice staff next received a personal thank-you card and a sweet treat, lifting spirits and promoting feelings of value. With staff engagement we then posed a question: 'What has COVID-19 taught you?' Another huge response showed that despite contending with morally traumatic situations, staff demonstrated support for their own psychological resilience in extremely challenging times. Feedback from this work was anonymised and displayed in the hospice, and staff were invited to view their work. The final step in this project was asking the team to share their hopes and wishes for the year ahead, displaying these to encourage our team to begin looking forward and feeling hope in a difficult time.

Results The results have been positive. A new feeling of openness and the ability to understand and reflect upon challenges of COVID-19 has contributed to a positive feeling in the hospice. The understanding that we all share common emotional responses is a powerful realisation, staff have shown remarkable bravery in sharing their moral injury.

Conclusion This project has made an impact on many members of the hospice team. Feeling safe to share feelings is essential to support resilience. It is vital that moving forward through the pandemic we continue wellbeing support and nurture self-care skills. 